\title{
Linear Polarization Features in the Quiet-Sun Photosphere: Structure and Dynamics
}

\author{
S. Kianfar ${ }^{1,2}(D)$ S. Jafarzadeh ${ }^{3,4}(\mathbb{D}) \cdot$ M.T. Mirtorabi $^{5}(D)$ \\ T.L. Riethmüller ${ }^{6}$
}

Received: 11 December 2017 / Accepted: 6 August 2018 / Published online: 23 August 2018

(C) The Author(s) 2018

\begin{abstract}
We present detailed characteristics of linear polarization features (LPFs) in the quiet-Sun photosphere from high-resolution observations obtained with SUNRISE/IMaX. We explore differently treated data with various noise levels in linear polarization signals, from which structure and dynamics of the LPFs are studied. Physical properties of the detected LPFs are also obtained from the results of Stokes inversions. The number of LPFs and their sizes and polarization signals are found to be strongly dependent on the noise level and on the spatial resolution. While the linear polarization with a signal-to-noise ratio $\geq 4.5$ covers about $26 \%$ of the entire area in the least noisy data in our study (with a noise level of $1.7 \times 10^{-4}$ in the unit of Stokes $I$ continuum), the detected (spatially resolved) LPFs cover about $10 \%$ of the area at any given time, with an occurrence rate on the order of $8 \times 10^{-3} \mathrm{~s}^{-1} \operatorname{arcsec}^{-2}$. The LPFs were found to be short lived (in the range of 30-300 s), relatively small structures (radii of $\approx 0.1-1.5$ arcsec), highly inclined, posing hG fields, and they move with an average horizontal speed of $1.2 \mathrm{~km} \mathrm{~s}^{-1}$. The LPFs were observed (almost) equally on both upflow and downflow regions, with an intensity contrast always larger than that of the average quiet Sun.
\end{abstract}

Keywords Magnetic fields · Photosphere: polarization · Optical

S. Kianfar

sepideh.kianfar@astro.su.se

1 Faculty of Basic Sciences, Azad University, P.O. Box 14676-86831, Tehran, Iran

2 Institute for Solar Physics, Department of Astronomy, Stockholm University, AlbaNova University Center, 10691 Stockholm, Sweden

3 Rosseland Center for Solar Physics, University of Oslo, P.O. Box 1029, Blindern, 0315 Oslo, Norway

4 Institute of Theoretical Astrophysics, University of Oslo, P.O. Box 1029, Blindern, 0315 Oslo, Norway

5 Department of Physics, Alzahra University, P.O. Box 1993893973, Tehran, Iran

6 Max Planck Institute for Solar System Research, Justus-von-Liebig-Weg 3, 37077 Göttingen, Germany 


\section{Introduction}

The magnetic field in the solar photosphere is often inferred by measuring different polarization states (i.e., Stokes parameters; e.g., Stenflo 1971, Wittmann 1974, Auer, Heasley, and House 1977), from observations of magnetically sensitive lines, such as Fe I $5250.2 \AA$. The magnetic structures are distributed all over the solar surface with a variety of temporal and spatial scales, and they have a wide range of inclination angles (with respect to the surface normal; Stenflo 2008, Martínez González et al. 2008, de Wijn et al. 2009, Solanki 2009, Sánchez Almeida and Martínez González 2011). Among these, a linear polarization signal (i.e., transverse component of the magnetic field) is ubiquitously found in active regions (Chae, Moon, and Pevtsov 2004, Kubo and Shimizu 2007, Cheung et al. 2008, Lites et al. 2008b), in quiet areas (Ishikawa and Tsuneta 2009, Lites et al. 2008b), but also in polar regions (Tsuneta et al. 2008) and at the solar limb (Martin 1988, Lites 2002).

Lites et al. (2017) reported the orientation of the magnetic fields in the quiet-Sun photosphere to be dominantly horizontal. However, the internetwork magnetic fields (Livingston and Harvey 1971, 1975, Martin 1988, Lin 1995, Lin and Rimmele 1999) have been diversely interpreted in the literature as mainly horizontal (Lites et al., 1996; Orozco Suárez et al., 2007; Ishikawa and Tsuneta, 2009; Orozco Suárez and Bellot Rubio, 2012), isotropic (Asensio Ramos, 2009; Bommier et al., 2009), or even predominantly vertical (Stenflo, 2010, 2013). Some of these interpretations (which are based on Stokes inversions) have been shown to be biased by, e.g., noise-affected Stokes parameters in the quiet Sun (Borrero and Kobel, 2011, 2012; Jafarzadeh et al., 2014; Borrero et al., 2017). We note that Stokes inversions return reliable inclination angles when applied to data with clear Stokes $Q$ and $U$ signal. It is also shown that conclusion between horizontal and quasi-isotropic distribution of the internetwork magnetic field is not straightforward, because the measurements are still limited by present telescopes in terms of, e.g., their spatial resolution and polarimetric accuracy, which prevent detection of magnetic properties of small-scale structures (for more information, see Lagg et al. 2016, Martínez González et al. 2016, Danilovic, van Noort, and Rempel 2016).

Early observations of the internetwork magnetic fields reported them as short-lived, horizontally inclined structures, typically smaller than $1^{\prime \prime}$ near the solar disk center (Lites et al. 1996), extending to a few arcseconds during their presence on the photosphere (De Pontieu 2002). These transient horizontal fields are reported to have strengths on the order of hG (Lites et al. 1996, Meunier, Solanki, and Livingston 1998). The nearly horizontal component of the magnetic fields, observed by Harvey et al. (2007), also shows seething patterns with variant spatial and temporal scales and an average linear polarization signal of about $10^{-3}$ in the unit of Stokes $I$ continuum $\left(I_{c}\right)$.

During the last decade, properties of the internetwork horizontal fields have been studied to higher degrees of accuracy using high-resolution observations with, e.g., the VTT (Beck and Rezaei 2009, Khomenko et al. 2003), Hinode (Lites et al. 2008a, Ishikawa and Tsuneta 2008, 2010, Jin, Wang, and Zhou 2009), and SUnRISE/IMaX (Danilovic et al. 2010). These studies have confirmed the transitory nature of the linear polarization features (LPFs) with $100-200 \mathrm{G}$ magnetic strength and typical lifetime and size of $1-10 \mathrm{~min}$ and a few arcsec, respectively; but also with features comparable to granular cells in both temporal and spatial scales (Ishikawa and Tsuneta 2010, 2011).

Horizontally inclined fields are found in both convective upflows and downflows, assumed to be compatible with horizontal magnetic flux (i.e., the magnetic flux of the horizontal magnetic field) whose advection to the surface is due to granular processes (De Pontieu 2002, Centeno et al. 2007, Orozco Suárez et al. 2007, Danilovic, van Noort, and 
Rempel 2016). Horizontal magnetic flux emerges inside a granule and is moved aside, to intergranular lanes, by plasma as the granule evolves (Stein and Nordlund 2006, Centeno et al. 2007, Ishikawa and Tsuneta 2008, Gömöry et al. 2010). The strong horizontal signal of the magnetic field in granules may manifest as the occurrence of low-lying magnetic loops (De Pontieu 2002, Martínez González et al. 2007, Gömöry et al. 2010, Jafarzadeh et al. 2017). They appear as linear polarization patches in the center of or above the granules (Lites et al. 2008a, Martínez González and Bellot Rubio 2009). The internetwork fields also tend to become more horizontal in the upper layers of the photosphere (Stenflo 2012, Danilovic, van Noort, and Rempel 2016). The horizontally oriented magnetic fields do not interfere in the granular evolution (Beck and Rezaei 2009), but the low-lying loops can rather reach the upper layers of the solar atmosphere; thus they may contribute to transient heating of the chromosphere and/or the corona (Schrijver et al. 1997, Lites et al. 2008b, Ishikawa and Tsuneta 2009).

Previously, the internetwork horizontal flux has been found to occur more frequently compared to the vertical internetwork field (Orozco Suárez et al. 2007, Lites et al. 2008b, Beck and Rezaei 2009, Orozco Suárez and Bellot Rubio 2012). However, recently the high spatial-resolution data from the SUNRISE 1-m balloon-borne solar telescope (Solanki et al., 2010; Barthol et al., 2011) has provided us with the largest LPF's rate of occurrence of $7 \times 10^{-4} \mathrm{~s}^{-1} \mathrm{acrsec}^{-2}$ (Danilovic et al., 2010), larger by $1-2$ orders of magnitude than previously reported.

In this study we aim to investigate the effect of the noise level on the number of identified LPFs, and hence, their statistical properties, namely, size, lifetime, linear polarization signal, proper motion, line-of-sight (LOS) velocity, field strength, inclination angle, and temperature, by extending the work of Danilovic et al. (2010). We provide a thorough overview of properties of LPFs in the quiet-Sun internetwork from high-resolution spectropolarimetric observations obtained with the Imaging Magnetograph eXperiment (IMaX; Martínez Pillet et al. 2011) on board the SUNRISE, and also from the results of Stokes inversions. We analyze differently treated images of the same data set (i.e., non-reconstructed, spatiallysmoothed non-reconstructed, and reconstructed; see Section 2) to identify features based on various signal-to-noise $(\mathrm{S} / \mathrm{N})$ ratios. The characteristics of the detected features are provided in Section 3, and the concluding remarks are presented in Section 4.

\section{Observations and Data Preparation}

We study a quiet-Sun area of $45^{\prime \prime} \times 45^{\prime \prime}$ at disk center acquired by SUNSRISE/IMaX on 2009 June 9 (between 01:32 and 01:58 UT), with sampling and temporal resolutions of $0.0545 \mathrm{arcsec} / \mathrm{pixel}$ and $33 \mathrm{~s}$, respectively. The effective field-of-view (FOV) under study was created after removing the edges of the original $51^{\prime \prime} \times 51^{\prime \prime}$ images, influenced by the apodization effect. The data set includes full Stokes $(I, Q, U$, and $V$ ) observations sampled in five wavelength positions ( \pm 80 and $\pm 40 \mathrm{~m} \AA$ in the line, and $+227 \mathrm{~m} \AA$ as the continuum) around the magnetically sensitive line Fe I centered at $5250.2 \AA$.

We aim at detecting LPFs (i.e., contiguous pixels with considerable linear polarization signals) in these quiet-Sun data, which largely include relatively weak polarization signals, particularly in the Stokes $Q$ and $U$ (Borrero and Kobel 2011, Danilovic, van Noort, and Rempel 2016). The less noisy the linear polarization maps become, the larger number of features, or features with larger sizes, are detected (i.e., the signals are uncovered to a larger extent; Orozco Suárez and Bellot Rubio 2012). Thus, to investigate the effect of noise in identification of these features, we inspect differently treated data of the same time-series with various levels of noise (see below). 
The data were prepared through a set of instrumental corrections for, e.g., temporal intensity fluctuations, interference fringes and dust particles in optical elements (including dark and flat-fielding), along with minimizing jitter-induced residual signal and instrument-caused blue-shifts (Martínez Pillet et al. 2011). These produced the so-called non-reconstructed data (NR; level 1), with $1 \sigma$ noise levels of $8.3 \times 10^{-4} I_{c}$ and $1.1 \times 10^{-3} I_{c}$ in Stokes $Q$ and $U$, respectively (Jafarzadeh et al., 2014). The noise levels of Stokes $Q$ and $U$ were determined as the standard deviations at the continuum positions of the corresponding Stokes parameter, since no significant polarization signal is expected in their continuum.

Furthermore, these data products were passed through a phase-diversity (PD) procedure according to the point-spread function (PSF) of the instrument/telescope which amplifies signal frequencies (i.e., PD-reconstructed data, PDR; level 2; Martínez Pillet et al. 2011). The latter procedure resulted in images with a higher spatial resolution (by a factor of 2; reaching $0.15-0.18$ arcsec) compared to the NR data, but also with a larger noise level (by a factor of $\approx 3$ ). The Stokes $Q$ and $U$ of the PDR data have noise levels of $2.6 \times 10^{-3} I_{c}$ and $3.6 \times 10^{-3} I_{c}$, respectively.

The linear polarization maps (we will refer to these images as transverse magnetograms) are then constructed from individual Stokes $Q$ and $U$ signals (from both NR and PDR data) at each wavelength position $i$ as $\sqrt{Q_{i}^{2}+U_{i}^{2}}$. The net linear polarization (LP; Lites et al. 2008b, Martínez Pillet et al. 2011) is then formed by averaging the four wavelength positions inside the Fe I 5250.2 A line, normalized to $I_{c}$ of each corresponding pixel,

$$
\mathrm{LP}=\frac{1}{4 I_{c}} \sum_{i=1}^{4} \sqrt{Q_{i}^{2}+U_{i}^{2}} .
$$

The LP has half of the noise of the linear polarization at any individual wavelength position $\left(\right.$ i.e., $\left.\sigma_{\mathrm{LPind}} / \sqrt{4}\right)$. We note that the noise level of individual wavelength positions $\left(\sigma_{\mathrm{LPind}}\right)$ was determined as the standard deviation of the linear polarization formed at the continuum position (i.e., $\left.\sigma \sqrt{Q_{\text {continuum }}^{2}+U_{\text {continuum }}^{2}}\right)$. Thus, $1 \sigma$ noise levels of $3.4 \times 10^{-4} I_{c}$ and $1.1 \times 10^{-3} I_{c}$ are obtained for $\mathrm{LP}$ constructed from NR and PDR data sets (i.e., $\mathrm{LP}_{\mathrm{NR}}$ and $\mathrm{LP}_{\mathrm{PDR}}$ ), respectively.

Figures 1 (a) and (b) display the LP maps of the first frame of NR and PDR data, respectively. The $\mathrm{LP}_{\mathrm{PDR}}$ illustrates sharper and smaller patches compared with that from NR data. Signal amplification of the PD reconstruction has increased the polarization signal by a factor of 2.5-3 compared to the one in the non-reconstructed map, but also had enhanced the noise level with about the same factor. We find that about the same number of pixels

Figure 1 Examples of LP maps (see main text) from the first frame of (a) non-reconstructed and (b) phase-diversity reconstructed data. The maps in panels (a) and (b) have $1 \sigma$ noise levels of $3.4 \times 10^{-4} I_{c}$ and $1.1 \times 10^{-3} I_{c}$, respectively.

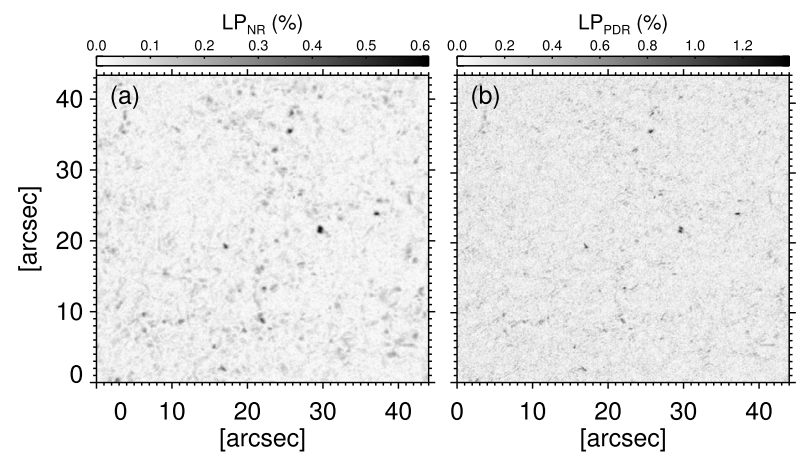


Table 1 Summary of $1 \sigma$ noise levels of linear polarization signals in differently treated data sets from SUNRISE/IMaX (PDR: phase-diversity reconstructed data; NR: non-reconstructed data; SSNR: spatially smoothed non-reconstructed data).

\begin{tabular}{llll}
\hline Parameter & PDR & NR & SSNR \\
\hline$Q / I_{C}$ & $2.6 \times 10^{-3}$ & $8.3 \times 10^{-4}$ & $4.6 \times 10^{-4}$ \\
$U / I_{C}$ & $3.6 \times 10^{-3}$ & $1.1 \times 10^{-3}$ & $4.8 \times 10^{-4}$ \\
LP & $1.1 \times 10^{-3}$ & $3.4 \times 10^{-4}$ & $1.7 \times 10^{-4}$ \\
\hline
\end{tabular}

pose signals greater than, or equal to, the noise level in both LP maps in Figure 1. Also, the $\mathrm{LP}_{\mathrm{NR}}$ includes only $14 \%$ more pixels with $\mathrm{S} / \mathrm{N} \geq 4.5$ than the $\mathrm{LP} \mathrm{PDR}_{\mathrm{PD}}$.

To additionally increase the $\mathrm{S} / \mathrm{N}$ in the linear polarization signals, similar to Jafarzadeh et al. (2014), we spatially smooth the non-reconstructed (SSNR) data by boxcar averaging of individual images (per Stokes parameter and per wavelength position) with a size of 3 pixels (i.e., averaging over 9 pixels without degrading the spatial resolution), prior to forming the LP. This smoothing results in single wavelength-position noise levels of $4.6 \times 10^{-4} I_{c}$ and $4.8 \times 10^{-4} I_{c}$ in Stokes $Q$ and $U$, respectively, and $1 \sigma$ noise level of $1.7 \times 10^{-4} I_{c}$, in $\mathrm{LP}_{\text {SSNR }}$. We note that the noise level of an LP is computed as the standard deviation of the corresponding linear polarization map computed for the continuum position, i.e., the standard deviation of $\sqrt{Q_{c}^{2}+U_{c}^{2}}$, where $Q_{c}$ and $U_{c}$ are the Stokes $Q$ and $U$ of the associated data set at the continuum position.

The latter noise level (i.e., from $\mathrm{LP}_{\mathrm{SSNR}}$ ) is the smallest in all the differently treated data sets introduced above, that is, smaller than those from the NR and PDR data by a factor of $\approx 2$ and $\approx 6.5$, respectively.

Figure 2 illustrates the effect of smoothing procedure on the S/N of a single LP map obtained from the first frame of data. Images in the right column show LP $_{\text {SSNR }}$ maps and the ones on the left represent the $\mathrm{LP}_{\mathrm{NR}}$ ones. The thresholds, as a factor of the corresponding noise levels, above which the LP signals are plotted are indicated on the images (i.e., labels in their lower left corners). As is visually evident in Figure 2, LPFs in smoothed maps are greater in both number and size, particularly in the second and third rows in Figure 2 (corresponding to a minimum $\mathrm{S} / \mathrm{N}$ of 3 and 4.5 , respectively). Pixels satisfying $\mathrm{S} / \mathrm{N} \geq 4.5$ cover about $26 \%$ of the entire LPSSNR map, which is larger by a factor of 2 than its nonsmoothed counterpart (i.e., the $\mathrm{LP}_{\mathrm{NR}}$ map). We note that a lower signal threshold results in a larger area covered by the linear polarization signal. However, the polarization signal is influenced, to a larger extent, by noise when pixels with smaller $\mathrm{S} / \mathrm{N}$ are included.

Table 1 summarizes the various noise levels of the differently treated data, discussed above. For comparison, we will search for LPFs in all the three treated data sets of LP $\mathrm{PDR}_{\mathrm{PD}}$, $\mathrm{LP}_{\mathrm{NR}}$, and LP $\mathrm{PSNR}_{\mathrm{SS}}$. However, the $\mathrm{LP}_{\mathrm{SSNR}}$ (with the lowest noise level in our sample) is considered as the primary data set, hence it provides the main results of this study.

\section{Analysis and Results}

We aim to search for LPFs in the LP maps from the three differently treated data sets (introduced in Section 2), and for the entire observing duration (consisting of 58 frames). The LPFs are defined as contiguous areas of minimum 10 pixels (i.e., the spatially resolved features) with considerable LP signals (i.e., greater than a specific noise level; Danilovic et al. 2010). We examine a variety of $\mathrm{S} / \mathrm{N}$, ranging from 1 to 6.5 , as the signal threshold, of which 


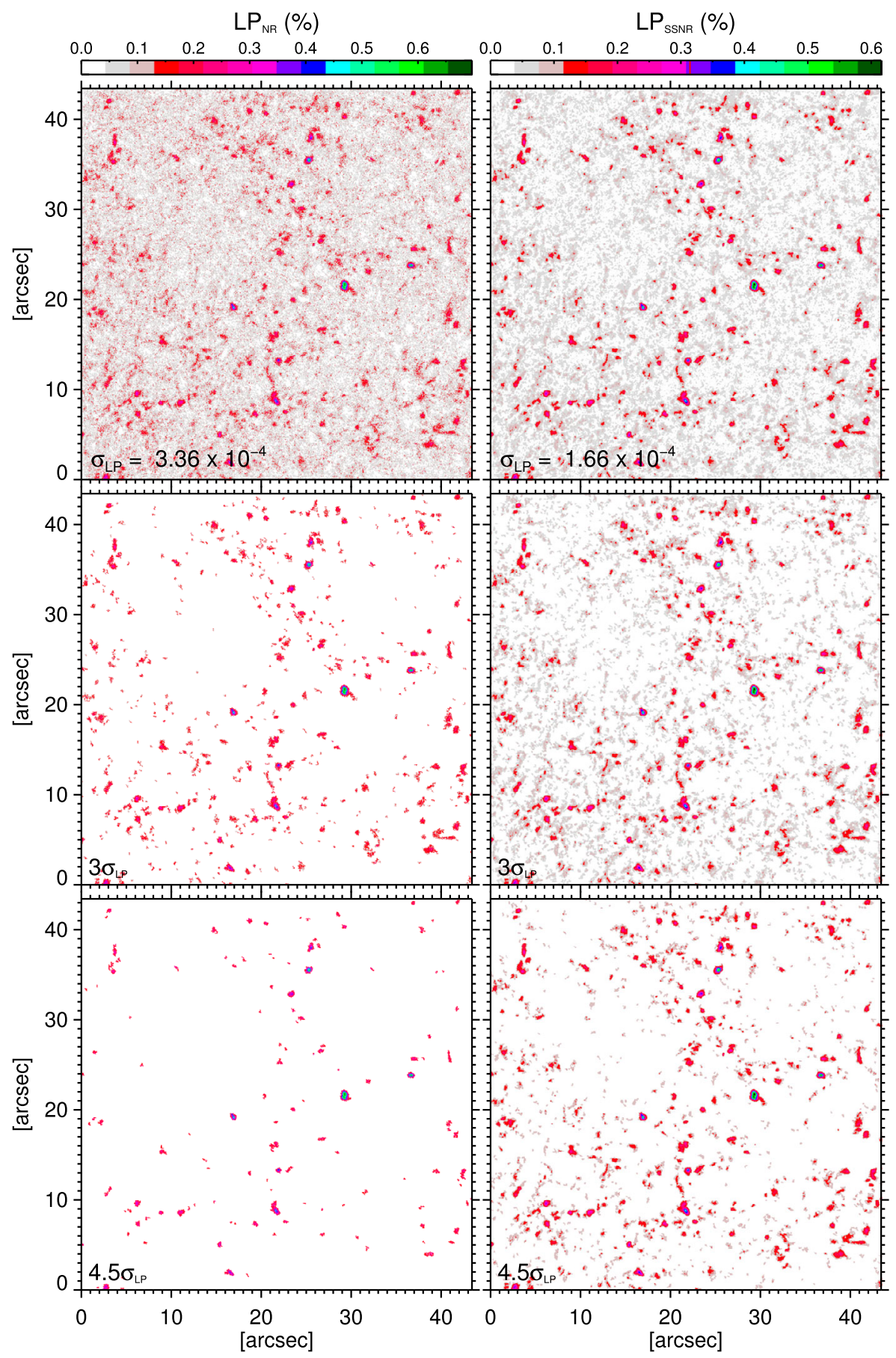

Figure 2 Examples of LP maps obtained from the first frame of non-reconstructed (left column) and spatially smoothed non-reconstructed (i.e., by boxcar averaging of $3 \times 3$ pixels; right column) data from SUNRISE/IMaX. From top to bottom, linear polarization signals above a certain threshold, labeled in the lower bottom corner of the panels, are plotted. 
$\mathrm{S} / \mathrm{N}=4.5$ is considered as the primary signal criterion since it results in a bigger number of features with precise boundaries.

The LPFs are found to be highly dynamic. During the course of their lifetimes, they face, at least, two of the following phenomena: (a) emergence, (b) submergence, (c) merging, and (d) splitting. Emergence and submergence of the patches are simply defined as rising and dropping of the signal above and below the noise level, respectively. It is noted that the features that are already emerged in the first frame are considered as new-born features. In addition, the ones that only appear in the last frame, are assumed to have a lifetime of only one frame. We also treat the product of both merging and splitting interactions as new-born features and consider parent features to be dead.

Properties of the identified LPFs (see below for the detection criteria), namely, size as well as average and maximum LP signals, are determined in individual frames, and the lifetime and horizontal velocity of the features are calculated in the time-series of LP images. We further obtain their other physical properties, such as magnetic-field strength, field inclination angle, LOS velocity, and temperature from the results of a Stokes inversion code. We provide distribution of the parameters and compare them by means of scatter plots between pairs of quantities.

All the above procedures are applied on the $\mathrm{LP}_{\mathrm{PDR}}, \mathrm{LP}_{\mathrm{NR}}$, and $\mathrm{LP}_{\mathrm{SSNR}}$ maps. Thus, we inspect the effect of noise level (or $\mathrm{S} / \mathrm{N}$ ) on the number of detected features, and consequently, on their determined properties.

\subsection{Detection and Tracking Procedures}

To detect LPFs in the differently treated LP maps, we employ an algorithm developed by Jafarzadeh, Rouppe van der Voort, and de la Cruz Rodríguez (2015). In detection procedure, the LP signal in a given LP map is cut at a certain signal threshold, which is defined as a factor of noise level (see Figure 2). Then, using the IDL routine blob_analyzer.pro (i.e., an algorithm for analyzing the regions of interest or the so-called "blobs" in an image), all contiguous remaining (non zero) pixels are identified and given an identity. Their properties, such as area (i.e., the number of pixels that each LPF includes), LP signal, and coordinates of the pixels within individual features are stored. In the detection process, the features are assumed to have circular shape. Therefore, LPFs smaller than a size threshold of 10 pixels, which leads to a diameter of $\approx 0.2 \operatorname{arcsec}$ (which is approximately equal to the resolution limit of the telescope), are excluded from the sample. Thus, the detected features are not noise originated, and they are spatially resolved.

We examine several signal thresholds on the LP maps (obtained by having been treated differently), ranging from $1 \sigma$ to $6.5 \sigma$, to find a proper $\mathrm{S} / \mathrm{N}$ which results in the greatest number of detected features that have the most precise boundaries and are not noise originated. An $\mathrm{S} / \mathrm{N} \geq 4.5$ is found to be the best choice. For comparison, we also present our analysis results for an $\mathrm{S} / \mathrm{N} \geq 3$, but, for simplicity, only for the $\mathrm{LP}_{\mathrm{NR}}$. The latter will show how the number and properties of detected features (of the same data set, and with the same noise level) depend on the threshold above which the LPFs are defined.

Furthermore, a new set of image sequences including only LPFs are created (i.e., areas outside the identified features are set to zero). These series of images are then used to track the LPFs in time. We use the same tracking algorithm as described by Jafarzadeh et al. (2013). In this method, the code locates each LPF by determining the center of gravity of its intensity as the position of the feature. Considering the location and size of the feature, a small area around each LPF is searched in consecutive frames. We note that only features above a certain signal threshold are included. Therefore, the "absent allowance" (where a 
Table 2 Statistics of the number of detected linear polarization features (LPFs) in differently treated data sets from SUNRISE/IMaX, containing signals above a given threshold.

\begin{tabular}{llclcl}
\hline $\begin{array}{l}\text { Data } \\
\text { set }\end{array}$ & $\begin{array}{l}\text { Signal } \\
\text { threshold }\end{array}$ & $\begin{array}{l}\text { Number of } \\
\text { LPFs per frame }\end{array}$ & $\begin{array}{l}\text { Area coverage } \\
\text { per frame }\end{array}$ & $\begin{array}{l}\text { Number of } \\
\text { individual LPFs }\end{array}$ & $\begin{array}{l}\text {Rate of occurrence }^{\mathrm{b}} \\
\left.\mathrm{s}^{-1} \operatorname{arcsec}^{-2}\right)\end{array}$ \\
\hline PDR & $4.5 \sigma_{\mathrm{LP}}$ & 544 & $0.3 \%$ & 1942 & $6.1 \times 10^{-4}$ \\
$\mathrm{NR}$ & $3 \sigma_{\mathrm{LP}}$ & 3479 & $5.0 \%$ & 14524 & $4.6 \times 10^{-3}$ \\
$\mathrm{NR}$ & $4.5 \sigma_{\mathrm{LP}}$ & 535 & $1.1 \%$ & 4092 & $1.3 \times 10^{-3}$ \\
$\mathrm{SSNR}$ & $4.5 \sigma_{\mathrm{LP}}$ & 2073 & $10.3 \%$ & 25099 & $7.9 \times 10^{-3}$ \\
\hline
\end{tabular}

a PDR: Phase-diversity reconstructed; NR: non-reconstructed; SSNR: spatially smoothed non-reconstructed.

${ }^{\mathrm{b}}$ When each LPF was counted once during its lifetime.

feature disappears in a few frames during the course of its lifetime, as a result of signal variation with time), which was considered for magnetic bright points in Jafarzadeh et al. (2013), is not performed here. Thus the tracking procedure returns lifetime and horizontal velocity of the LPFs, in addition to the LP signal, size and location of the features which were already stored in the detection phase.

Table 2 summarizes the average number of detected LPFs per frame (i.e., an FOV of $\left.45^{\prime \prime} \times 45^{\prime \prime}\right)$, the mean fraction of area covered by all the detected LPFs in each frame, and the number of individual LPFs tracked in the entire time-series of LP images, consisting of 58 frames (i.e., when each LPF is counted once during the course of its lifetime). These statistics are listed for the differently treated data sets. The signal thresholds, above which the LPFs are identified, are also specified.

The number of individual LPFs found in the SSNR maps are, on average, four times larger than those detected in the NR images, when the same signal threshold of $4.5 \sigma_{\mathrm{LP}}$ (of the corresponding data) is applied. Note that the total fraction of area covered by the detected LPFs in the LPSSNR are larger by an order of magnitude than those found in the $\mathrm{LP}_{\mathrm{NR}}$. The number of LPFs in the $\mathrm{LP}_{\mathrm{NR}}$ are comparable to those found in the noisier (but with a higher spatial resolution) $\mathrm{LP}_{\mathrm{PDR}}$ maps. The fraction of FOV covered by all the LPFs in one frame are, however, smaller, by a factor of 3 , in the latter data set. These may imply the effect of both spatial resolution and noise level on the number of identified features. Although in NR data several LPFs are detected as one feature, while they are identified individually resolved and separated in the $\mathrm{LP}_{\mathrm{PDR}}$ images in the case of applying the same signal threshold, i.e., $4.5 \sigma$, the $\mathrm{LP}_{\mathrm{NR}}$ images have a larger $\mathrm{S} / \mathrm{N}$, which results in a larger amount of identified signals (and hence, a larger number of individual features) compared with those from the LP PDR maps (see Table 2).

A comparison between the number of LPFs identified in the $\mathrm{LP}_{\mathrm{NR}}$ images with minimum $3 \sigma_{\mathrm{LP}}$ and $4.5 \sigma_{\mathrm{LP}}$ noise levels clarifies that the lower the signal threshold becomes, the larger number of features (and the bigger areal coverage) is obtained. Although the number

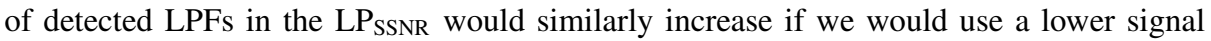
threshold of, e.g., $3 \sigma_{\mathrm{LP} S \mathrm{SSR}}$, the final results could be biased by the effect of noise. Clearly, the number of detected LPFs is a trade-off between the necessity of high $\mathrm{S} / \mathrm{N}$ of the data and the threshold with which the features are defined. Thus, we rather choose a conservative signal threshold of $4.5 \sigma$ in our analysis. We note that our size threshold of 10 pixels has limited the number of detected LPFs, hence, a lower limit of their rates of occurrence are obtained. This has, however, resulted in studying the spatially resolved features. 

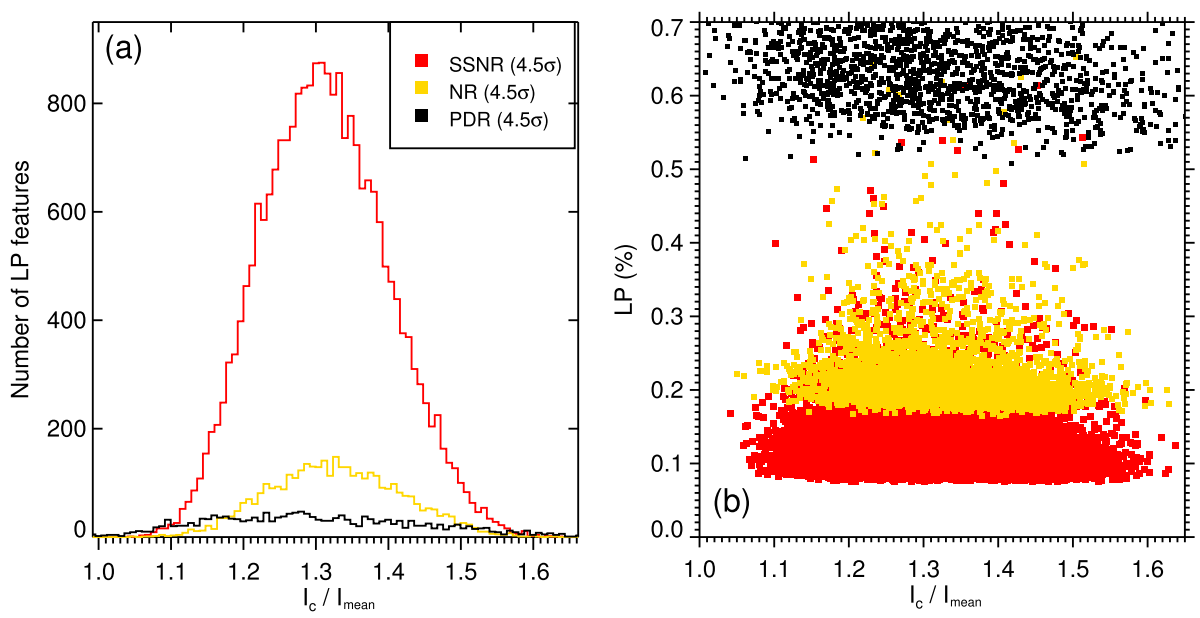

Figure 3 Distribution of intensity contrast of the detected LPFs (a), and mean linear polarization of the LPFs as a function of their contrast (b). The results from three differently treated data sets (PDR: Phase-diversity reconstructed data; NR: Non-reconstructed data; SSNR: Spatially smoothed non-reconstructed data) are plotted.

\subsubsection{Intensity Distribution}

Distributions of the mean intensity contrast of all detected LPFs from Stokes $I$ continuum (normalized to the average quiet Sun) from SSNR, NR, and PDR images are illustrated in Figure 3(a). These histograms show that the LPFs are all brighter than the average quiet Sun $\left(I_{c}=1\right)$. The relationships between these intensity contrast values and their mean LP signals are also plotted in Figure 3(b). The latter shows no relationship between the brightness and LP signal of the features under study.

\subsubsection{Physical and Dynamical Properties}

The LP signal, size, horizontal velocity, and lifetime of the detected LPFs from the differently treated data (see Table 2) were determined during the detection and tracking procedures. The distributions of these parameters are shown in Figure 4, and the relationships between pairs of the physical quantities are plotted in Figure 6. Below, these parameters are individually discussed in detail.

LP Signal The LP values of all pixels within the area of an LPF is extracted from the LP maps, i.e., the pixel values of the detected features are individually stored. The mean and maximum LP values of each LPF are calculated for further statistics, and the relationship is studied between the various parameters. The histograms of mean LP signals (Figure 4(a)) are all skewed to greater signal values. It is evident that by increasing the noise level, only features with stronger signals are detected. A comparison between the different histograms in Figure 4(a) also shows the effect of $\mathrm{S} / \mathrm{N}$ on the number of the identified LPFs. The distribution of the mean LP signals of the features found in the $\mathrm{LP}_{\mathrm{SSNR}}$ (i.e., the least noisy data set) ranges between $5 \sigma_{\mathrm{LP}} \mathrm{SSNR}$ and $11 \sigma_{\mathrm{LP}}{ }_{\mathrm{SSNR}}$, peaking at $\approx 8 \sigma_{\mathrm{LPSSN}}\left(\right.$ i.e., $1.3 \times 10^{-3} I_{c}$ ). 

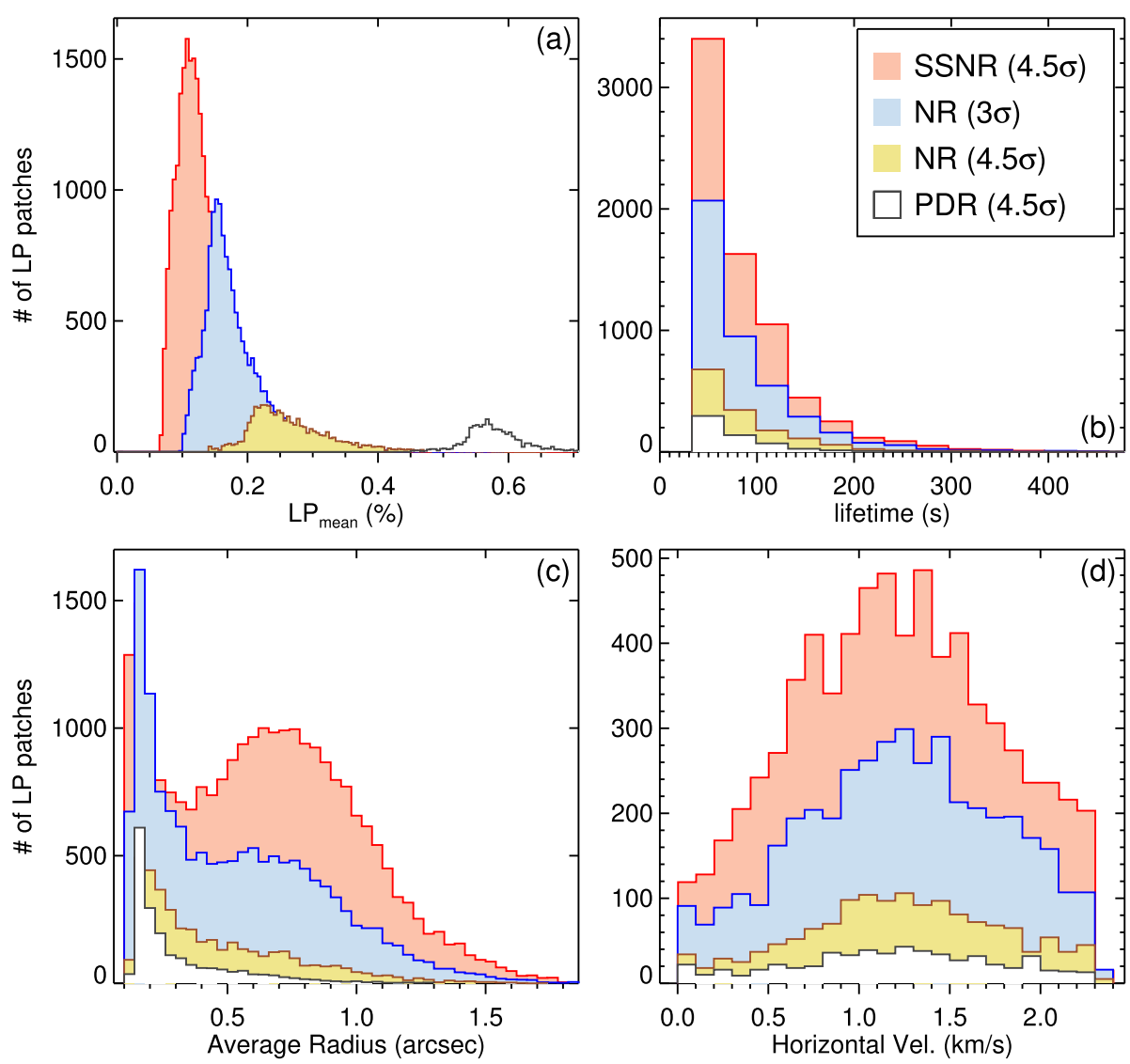

Figure 4 Distributions of mean LP signal (a), lifetime (b), average size (radius; (c)), and horizontal velocity (d) of the detected linear polarization features from differently treated data sets, i.e., PDR, NR and SSNR data with noise levels $(\sigma)$ of $1.1 \times 10^{-3}, 3.4 \times 10^{-4}$ and $1.7 \times 10^{-4}$ in terms of continuum intensity, respectively (see main text).

Lifetime About 65\%, in SSNR and NR data, and 75\%, in PDR data, of the total detected features are observed in only one frame (i.e., they have a maximum lifetime of about $33 \mathrm{~s}$ ). The lifetime of the rest of the other features are found from the tracking procedure, i.e., the time difference between the detected feature in the last frame and the first one. Figure 4(b) shows the lifetime distribution of the LPFs which live longer than one frame. Thus the histograms are limited at $33 \mathrm{~s}$ in their lower ends.

These histograms indicate that lifetimes of the most of the LPFs that live longer than one frame range between 1 and $8 \mathrm{~min}$. The lifetimes of the LPFs show no significant correlation with the $\mathrm{S} / \mathrm{N}$. The average lifetime in all the data sets is about $70 \mathrm{~s}$. However, the highest signal-to-noise ratio in SSNR data leads to the highest fraction (35\%) of long-lived features (i.e., features that live more than $8 \mathrm{~min}$ ) among all tracked patches. This may suggest that the relatively weak LP signals at the beginning and/or at the end of the LPFs' life are being uncovered to a rather large extent when they are detected in images with larger $\mathrm{S} / \mathrm{N}$. 

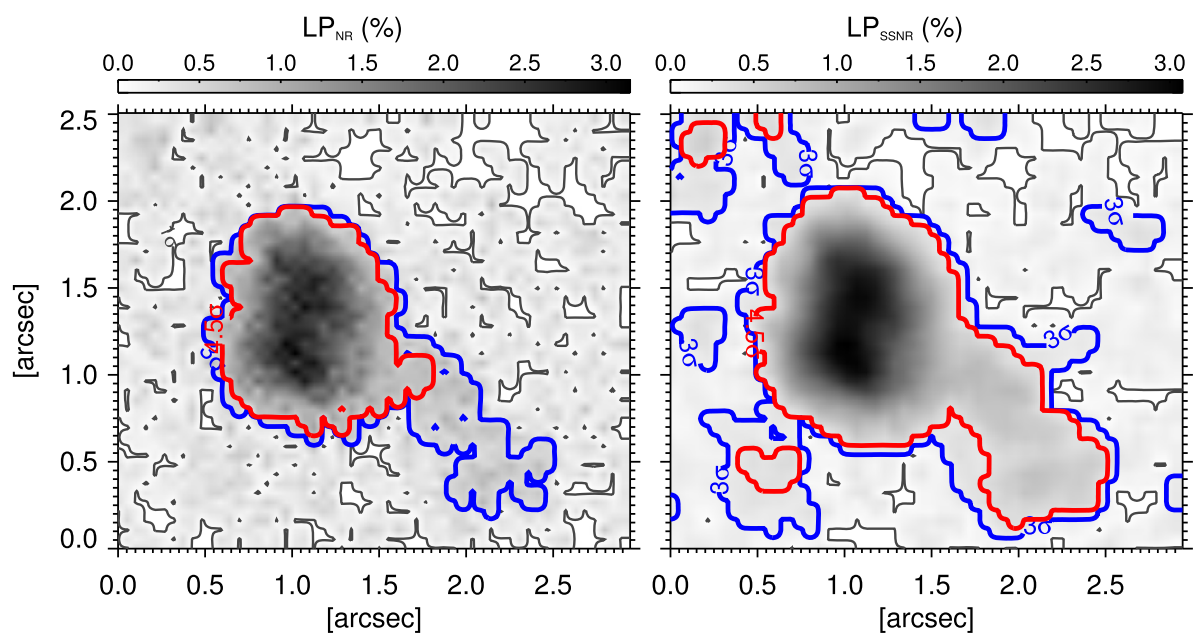

Figure 5 A linear polarization feature (LPF) in non-reconstructed (NR; left) and spatially smoothed non-reconstructed (SSNR; right) images. The black, blue and red contours mark the corresponding $1 \sigma_{\mathrm{LP}}, 3 \sigma_{\mathrm{LP}}$, and $4.5 \sigma_{\text {LP }}$ noise levels (see Table 2). The differences between size and number of the detected features in the two panels are evident.

Size The size (i.e., the cross-section) of an LPF is defined as the number of pixels that are included in a feature boundary, defined based on a certain signal threshold. Figure 4(c) shows the distribution of mean radius, computed from their areas (by assuming a circular shape). The LPFs in all the four data sets appear to have radius in the order of $0.1-1.5 \operatorname{arcsec}$, comparable to the size of granules. It is also found that the higher $\mathrm{S} / \mathrm{N}$ uncovers a larger (hidden) part of the LPFs, thus a larger number of features with bigger sizes are found in the SSNR data compared to the other (noisier) data sets. It is similarly the case when comparing the size distribution of $\mathrm{LPF}_{\mathrm{NR}}$ with $\mathrm{S} / \mathrm{N}$ threshold of 3 and 4.5, the former includes many more large features.

The larger size of features revealed by higher $\mathrm{S} / \mathrm{N}$ (or lower signal threshold) may indicate that the LP signal radially reduces from the center of the LPFs toward the edges of the patches. Figure 5 illustrates the effect of these two factors on the size of a typical patch. The detected feature with the signal threshold of $4.5 \sigma_{\mathrm{LP}}$ (red contour) is smaller in the NR map (left panel), compared to that found in the SSNR image (right panel). Also, a similar size difference is found for a lower signal threshold of $3 \sigma_{\mathrm{LP}}$, shown with the blue contour. The LPF detected with the latter signal threshold encompasses a larger region than the case of applying $4.5 \sigma_{\mathrm{LP}}$ threshold on the same map. In addition, the $\mathrm{LP}_{\mathrm{SSNR}}$ includes more than one isolated feature at both signal thresholds of $3 \sigma_{\mathrm{LP}}$ and $4.5 \sigma_{\mathrm{LP}}$ (i.e., the separated islands shown with the blue and red contours, respectively), whereas only one feature is identified in the $\mathrm{LP}_{\mathrm{NR}}$ image.

Horizontal Velocity The instantaneous horizontal velocity is determined for the tracked features (i.e., the features that lived for more than one frame). It is obtained by dividing the distance that each LPF moves between consecutive frames by their observing time difference (i.e., the cadence of the observations; $33 \mathrm{~s}$ ).

We note that the tracking algorithm considers a feature to be the same as in the previous frame if it is spatially located in a small area around it. This condition has inevitably led to an upper limit for the determined horizontal velocities. Although this criterion may exclude 

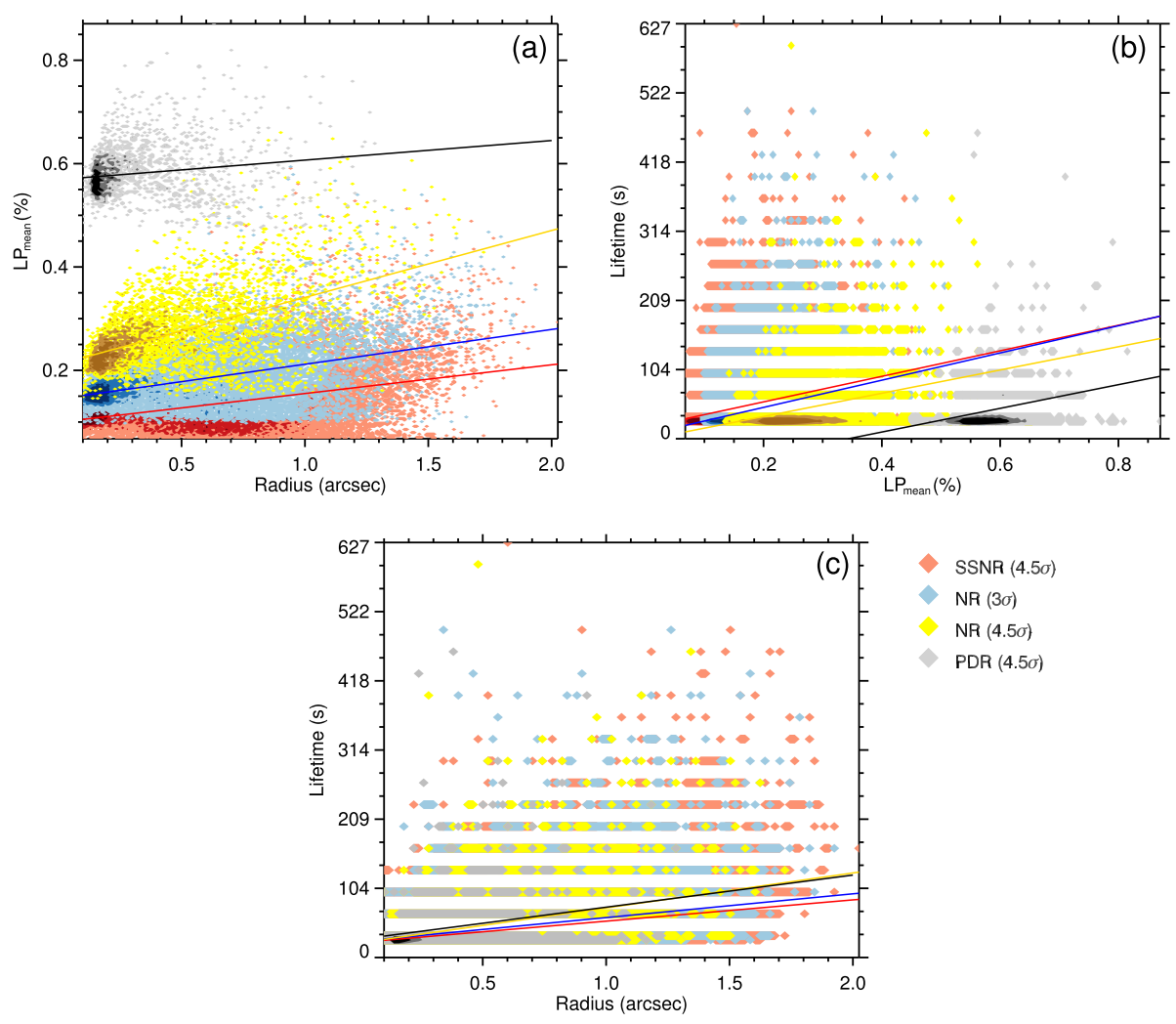

$$
\begin{aligned}
& \text { SSNR }(4.5 \sigma) \\
& \text { NR }(3 \sigma) \\
& \text { NR }(4.5 \sigma) \\
& \text { PDR }(4.5 \sigma)
\end{aligned}
$$

Figure 6 Relationships between various pairs of parameters of the LPFs, detected in differently treated data sets (see main text).

the very fast-moving features, it has secured our detection from mixing of the apparently close features. In addition, the horizontal velocity has been only measured for the LPFs whose lifetimes are longer than $33 \mathrm{~s}$ (i.e., when they are observed, at least, in two consecutive frames).

Figure 4(d) shows the distribution of horizontal velocity of the detected LPFs from the differently treated data sets. The four histograms show a nearly normal distribution. They all range between $0-2.4 \mathrm{~km} \mathrm{~s}^{-1}$, and peak at a nearly same mean velocity of $1.2 \mathrm{~km} \mathrm{~s}^{-1}$.

Figure 6 deisplays the scatter density plots of the various parameters against each other. The points with darker colors show densely scattered areas. In Figure 6(a) the distribution of mean LP of the tracked LPFs are plotted with respect to their mean radius, averaged over the course of their lifetimes. The linear fits to the data points show a direct correlation between the $\mathrm{LP}_{\text {mean }}$ and mean radius of the LPFs in all the four differently treated data sets.

A direct correlation also exists in the case of $\mathrm{LP}_{\text {mean }}$ versus lifetime and the average radius versus lifetime, respectively, shown in Figure 6(b) and (c). The lifetime scatter plots in Figure 6 are binned to the cadence of the observations, i.e., $33 \mathrm{~s}$.

These correlations indicate that the bigger features pose, on average, larger LP signals and tend to live longer on the solar surface. 
No clear relationships between the horizontal velocity of the LPFs and their other properties was found.

\subsection{Stokes Inversion}

We also use the results of Stokes inversion (of the PDR data) to obtain additional physical properties of the detected LPFs. These include LOS velocity, magnetic field strength, field inclination, and temperature. We use the results of the SPINOR inversion code (Solanki, 1987; Frutiger et al., 2000; Berdyugina, Solanki, and Frutiger, 2003). The code used the Harvard Smithsonian Reference Atmosphere (HSRA; Gingerich et al. 1971) as the initial model atmosphere, and performed height-dependent computation of the temperature at three nodes along the depth scale (at $\log \left[\tau_{500 \mathrm{~nm}}\right]=0,-0.9$, and -2.5 ). The other parameters were calculated at one node. For details on the code and the specifications applied on the same data see Kahil, Riethmüller, and Solanki (2017). It is noted that the inversion code have assumed a unity magnetic filling factor.

We extract the physical parameters from the corresponding pixels (of the center of gravity of LP signal) of the LPFs from the results of the Stokes inversion. Although these may not represent the physical properties of all pixels across individual LPFs, the parameters correspond to pixels with the largest $\mathrm{S} / \mathrm{N}$ in each feature.

\subsubsection{Physical Properties from Stokes Inversion}

The distributions of the magnetic field strength, field inclination, and the LOS velocity of the LPFs, from the SPINOR inversion code, are plotted in Figure 7(a)-(c), respectively. They show that the magnetic field at the position of LPFs are almost horizontal, with a rather narrow histogram peaking at an inclination angle of 90 degrees (Figure 7(b)). The LPFs are found to pose hG fields at their center of gravity (Figure 7(a)). We note, however, that the field strength has been likely underestimated at individual pixels (they are spatially unresolved). The histogram of LOS velocity of the LPFs, shown in Figure 7(c), demonstrates that they are located on both the center of the granules, associated to upflows, and the granular edges, where downflows have been reported (Roudier et al. 2001, Nordlund, Stein, and Asplund 2009). Their distributions over the two regions are nearly equal in the results of the SPINOR code.

The distributions of the temperature at the position of the detected LPFs are plotted at three optical depths in Figure 8. We found that the LPFs were, on average, brighter than the
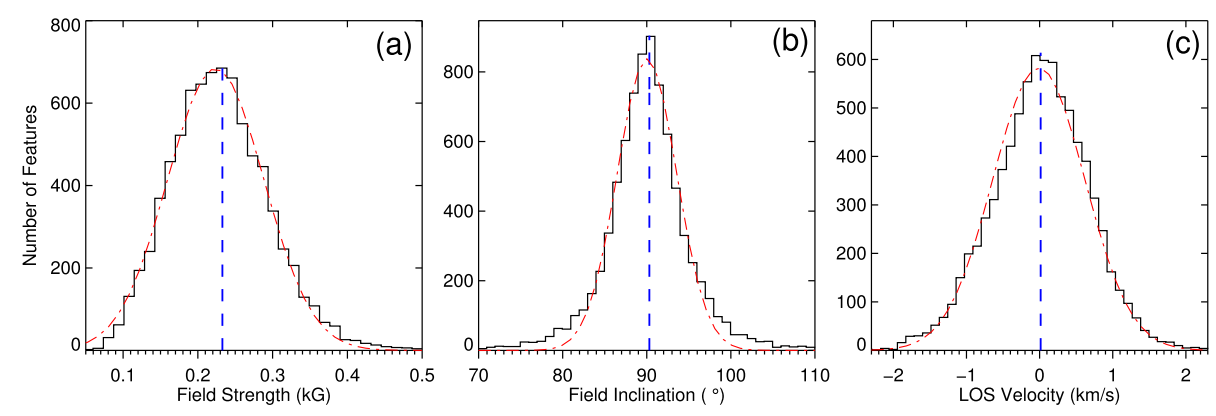

Figure 7 Distributions of the field strength (a), field inclination (b), and LOS velocity of the LPFs from the PDR Stokes inversion with the SPINOR code. 


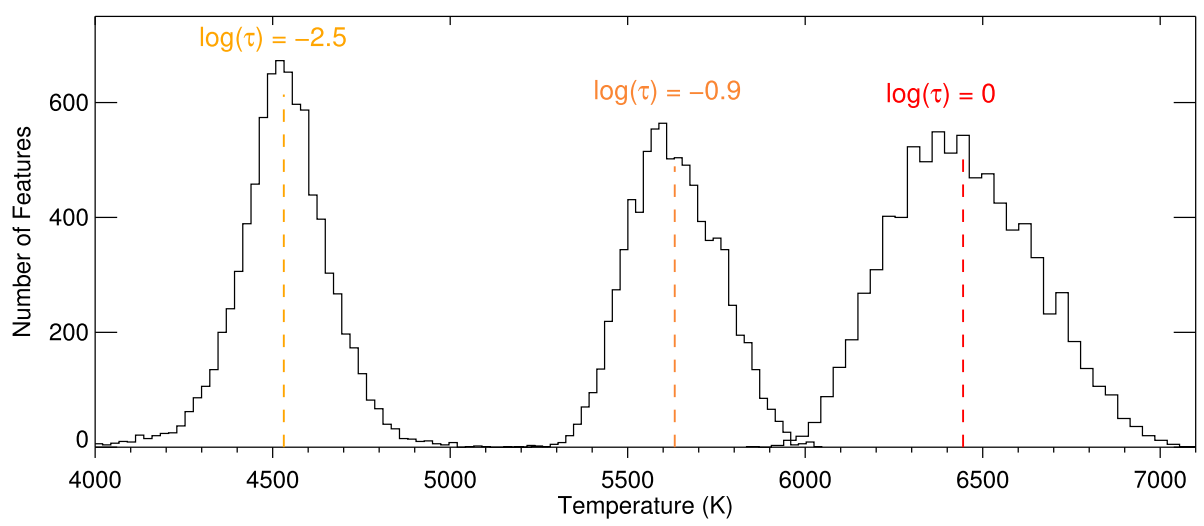

Figure 8 Distributions of the temperature at the location of the LPFs, from the results of the SPINOR inversion code. The distributions at three optical depths (labeled above the peak of each histogram) are plotted.
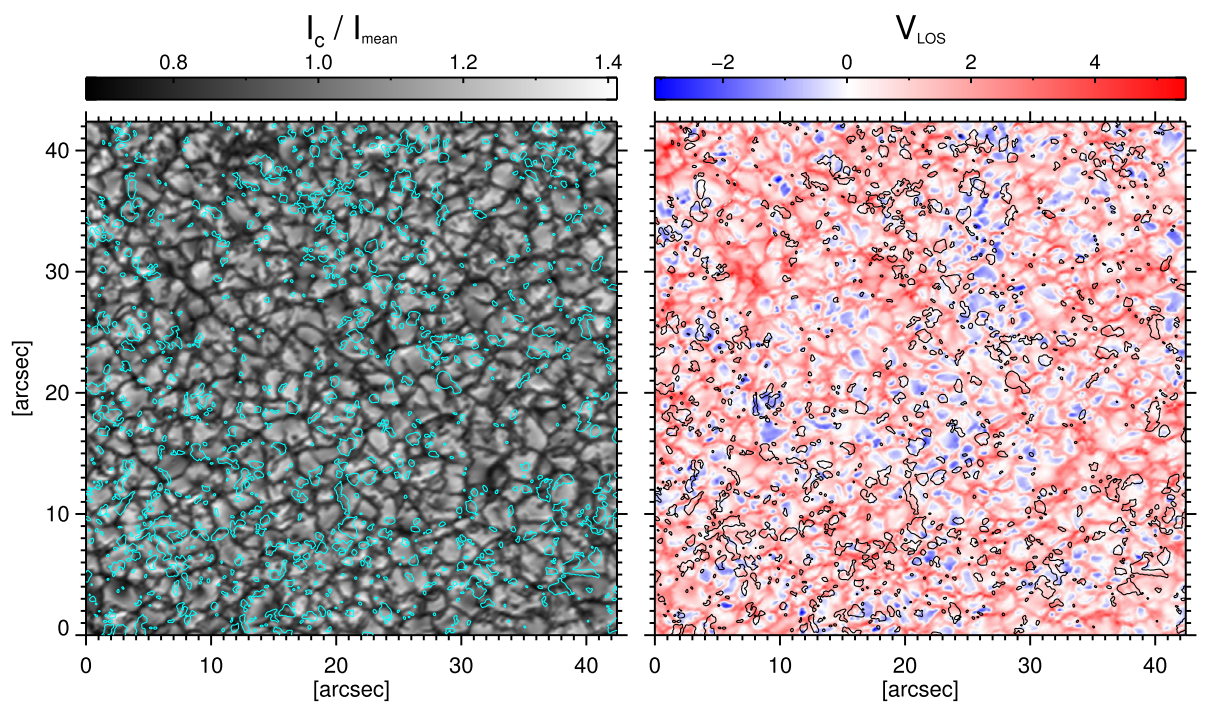

Figure 9 Spatial locations of the linear polarization features (LPFs) on a continuum intensity image (left), and on its corresponding LOS velocity map (right) of the first frame of PDR data set.

quiet Sun (see Section 3.1.1). This implies that the LPFs are likely located over the solar granules. This agrees with the decrease of their temperatures, on average, with height (see Figure 8), which is expected from the standard model atmosphere, such as FALC (Fontenla et al., 2006).

To also visually inspect the locations of the LPFs with respect to the granulation pattern, we have plotted the identified LPFs on a continuum intensity image and its corresponding LOS velocity map in the left and right panels of Figure 9, respectively. These show that the LPFs are mostly located over the granules. A small fraction of some of the LPFs seems to be extended to the intergranular areas. 


\section{Conclusions}

We have carried out a thorough study of (statistical properties of) linear polarization features (LPFs) by exploiting the high spatial- and temporal-resolution observations of the quiet-Sun internetwork, obtained with SUNRISE/IMaX. The LPFs are defined as areas with at least 10 contiguous pixels whose LP signals are above a particular threshold level. We inspected the effect of the $\mathrm{S} / \mathrm{N}$ in our study by analyzing the LPFs in three differently treated data sets (with different noise levels; see Table 1) and applying various signal thresholds.

We found a total number of $\approx 25100$ individual LPFs (when each LPF was only counted once during the course of its lifetime) during the 31 min time-series of images of the SSNR data (i.e., consisting 58 frames of $45^{\prime \prime} \times 45^{\prime \prime}$ ). This is, to the best of our knowledge, the largest number of individual LPFs found so far, with the rate of occurrence on the order of $8 \times 10^{-3} \mathrm{~s}^{-1} \operatorname{arcsec}^{-2}$. This rate of occurrence is larger, by an order of magnitude, than that of found by Danilovic et al. (2010). We should, however, note that the rate of occurrence strongly depends on the definition of the features under study. The detected LPFs from the SSNR images were found to have average radii on the order of $0.5-1 \operatorname{arcsec}$ and cover about $10.3 \%$ of the quiet-Sun internetwork.

Spatially smoothing of the NR data, resulting in a higher $\mathrm{S} / \mathrm{N}$, resulted in the detection of larger LPFs. The phase-diversity reconstructed data, leading to a higher spatial resolution compared to the NR images, resulted in identification of very small LPFs with an average diameter of $\approx 0.2 \operatorname{arcsec}$. The $\mathrm{S} / \mathrm{N}$ were also found to influence the distribution of lifetime of the LPFs, so longer-lived features were detected in data sets with higher S/N. The lifetime distributions of the LPFs (peaking at about $1 \mathrm{~min}$ ) drop exponentially for all the differently treated data sets. The short lifetimes of our LPFs are in agreement with the transient nature of these features reported by Lites et al. (1996) and Danilovic et al. (2010), but they are smaller than the average lifetime obtained by De Pontieu (2002), Ishikawa and Tsuneta (2008) and Jin, Wang, and Zhou (2009). The lifetimes of our LPFs are found to be correlated with their both mean LP signals and mean sizes (when averaged over their lifetimes).

The LPFs in our study horizontally move with an average speed of $1.2 \mathrm{~km} \mathrm{~s}^{-1}$, with nearly normal distributions ranging between $0-2.5 \mathrm{~km} \mathrm{~s}^{-1}$. We found no correlation between the $\mathrm{S} / \mathrm{N}$ of the employed data sets and the distributions of horizontal velocity of the LPFs.

We also examined the effect of signal threshold on the NR data. With a threshold of $4.5 \sigma_{\mathrm{LP}}$, we found the smallest number of detected LPFs per frame (535 features on average) which occupies only $1.1 \%$ of the total area. These LPFs emerge with a size of $\approx 0.6$ arcsec and a minimum LP signal of about $2.7 \times 10^{-3} I_{c}$. They reach a peak size and LP SIGNAL of 0.7 arcsec and $2.9 \times 10^{-3} I_{c}$ during their lifetimes, respectively, and disappear with almost having the same sizes and LP signals as the moment of their appearance on the solar surface. A total number of 4092 individual LPFs were tracked in the NR time-series of images, among which, about $25-35 \%$ lived longer than $33 \mathrm{~s}$ (i.e., they were tracked in more than one frame). Applying a lower signal threshold of $3 \sigma_{\mathrm{LP}}$ lead to a greater number of detected features in each frame, compared to those found with the signal threshold of $4.5 \sigma_{\mathrm{LP}}$. These LPFs (in NR images) cover about $5 \%$ of the area, with the percentage of the number of features living longer than $33 \mathrm{~s}$ equal to $29 \%$. Due to the lower signal threshold, the weaker features were also detected. These features have smaller mean LP signal than that of found for the LPFs with a signal threshold of $4.5 \sigma_{\mathrm{LP}}$, while the sizes of the former are, on average, bigger than the latter. This indicates that the LP signal decreases from the center of the patches toward their boundaries, which can, however, be a result of the limited spatial resolution of the data set. 
We also inspected the effect of the spatial resolution by investigating the LPFs in the PDR maps (i.e., with a larger spatial resolution, by a factor of 2, compared to the NR images), which also has the largest noise level in our samples due to the reconstruction process (which amplifies the signal and the noise at the same time; see Table 1). The LPFs studied in this data set (with a signal threshold of $4.5 \sigma_{\mathrm{LP}}$ ) have an average LP signal of $\approx 3.9 \times 10^{-3} I_{c}$. They were found to be, on average, the shortest lived and the smallest features among the LPFs detected in all the other data sets.

We found a weak but positive correlation (due to the large scatter shown in Figure 6) between the LP signal, size and lifetime of the features in all SSNR, NR, and PDR images. The Pearson correlation coefficient $(r)$ for the scatter density plots shown in Figure 6, has been calculated for the most populated sample (i.e., the SSNR LPFs) as

$$
r=\frac{\sum_{i=0}^{n}\left(x_{i}-\bar{x}\right)\left(y_{i}-\bar{y}\right)}{\sqrt{\sum_{i=0}^{n}\left(x_{i}-\bar{x}\right)^{2}} \sqrt{\sum_{i=0}^{n}\left(y_{i}-\bar{y}\right)^{2}}},
$$

where $x$ and $y$ represent the properties plotted in each panel of Figure 6 and $n$ is the number of individual tracked LPFs in SSNR data. The coefficient values for the scatter plots of $\mathrm{LP}_{\text {mean }}$-radius (Figure 6(a)), lifetime-LP $\mathrm{P}_{\text {mean }}$ (Figure 6(b)) and lifetime-radius (Figure 6(c)) are $+0.41,+0.59$ and +0.37 , respectively. In other words, the LPFs with larger LP signal tend to grow to larger sizes and to live longer, whereas the relatively small features with relatively weak LP signal are more transient. The horizontal velocity with which features move along the surface shows no correlation with the other parameters in either of the differently treated data sets.

Furthermore, from the results of a Stokes inversion of the PDR data, we found that the LPFs have a magnetic field strength in the range of $50-500 \mathrm{G}$, peaking at $230 \mathrm{G}$. This agrees with the hG horizontal magnetic fields reported by Lites et al. (1996) and Meunier, Solanki, and Livingston (1998). The mainly horizontal orientation of LPFs in the photospheric IN regions (Livingston and Harvey 1971, 1975, Martin 1988, Lin 1995, Lin and Rimmele 1999, Lites et al. 2017) was observed as the predominant inclination of $90^{\circ}$, in a range of 70 to 110 degrees. On average, the temperature at the location of the LPFs decreases with height in the solar photosphere.

Interactions between the LPFs, such as merging and splitting, and variations of the properties of the LPFs with time were not investigated here and are the subject of a future study.

Acknowledgements We are grateful to A. Lagg for detailed and helpful comments on the manuscript. S.K. acknowledges the support from CHROMATIC project (2016.0019) funded by the Knut and Alice Wallenberg foundation. S.J. acknowledges support from the European Research Council (ERC) under the European Union's Horizon 2020 research and innovation program (grant agreement No. 682462) and from the Research Council of Norway through its Centres of Excellence scheme, project number 262622. M.T.M. acknowledges support from the Iranian National Research Institute for Science Policy, Ministry of Higher Education. The German contribution to SUNRISE is funded by the Bundesministerium für Wirtschaft und Technologie through Deutsches Zentrum für Luft- und Raumfahrt e.V. (DLR), grant No. 50 OU 0401, and by the Innovationsfond of the President of the Max Planck Society (MPG). The Spanish contribution has been funded by the Spanish MICINN under projects ESP2006-13030-C06 and AYA2009-14105-C06 (including European FEDER funds). The HAO contribution was partly funded through NASA grant NNX08AH38G.

Disclosure of Potential Conflicts of Interest The authors declare that they have no conflicts of interest.

Open Access This article is distributed under the terms of the Creative Commons Attribution 4.0 International License (http://creativecommons.org/licenses/by/4.0/), which permits unrestricted use, distribution, and reproduction in any medium, provided you give appropriate credit to the original author(s) and the source, provide a link to the Creative Commons license, and indicate if changes were made. 


\section{References}

Asensio Ramos, A.: 2009, Evidence for quasi-isotropic magnetic fields from hinode quiet-sun observations. Astrophys. J. 701, 1032. DOI. ADS.

Auer, L.H., Heasley, J.N., House, L.L.: 1977, The determination of vector magnetic fields from Stokes profiles. Solar Phys. 55, 47. DOI. ADS.

Barthol, P., Gandorfer, A., Solanki, S.K., Schüssler, M., Chares, B., Curdt, W., Deutsch, W., Feller, A., Germerott, D., Grauf, B., Heerlein, K., Hirzberger, J., Kolleck, M., Meller, R., Müller, R., Riethmüller, T.L., Tomasch, G., Knölker, M., Lites, B.W., Card, G., Elmore, D., Fox, J., Lecinski, A., Nelson, P., Summers, R., Watt, A., Martínez Pillet, V., Bonet, J.A., Schmidt, W., Berkefeld, T., Title, A.M., Domingo, V., Gasent Blesa, J.L., del Toro Iniesta, J.C., López Jiménez, A., Álvarez-Herrero, A., Sabau-Graziati, L., Widani, C., Haberler, P., Härtel, K., Kampf, D., Levin, T., Pérez Grande, I., Sanz-Andrés, A., Schmidt, E.: 2011, The Sunrise Mission. Solar Phys. 268, 1. DOI. ADS.

Beck, C., Rezaei, R.: 2009, The magnetic flux of the quiet Sun internetwork as observed with the Tenerife infrared polarimeter. Astron. Astrophys. 502, 969. DOI. ADS.

Berdyugina, S.V., Solanki, S.K., Frutiger, C.: 2003, The molecular Zeeman effect and diagnostics of solar and stellar magnetic fields, II: Synthetic Stokes profiles in the Zeeman regime. Astron. Astrophys. 412, 513. DOI. ADS.

Bommier, V., Martínez González, M., Bianda, M., Frisch, H., Asensio Ramos, A., Gelly, B., Landi Degl'Innocenti, E.: 2009, The quiet Sun magnetic field observed with ZIMPOL on THEMIS, I: The probability density function. Astron. Astrophys. 506, 1415. DOI. ADS.

Borrero, J.M., Kobel, P.: 2011, Inferring the magnetic field vector in the quiet Sun, I: Photon noise and selection criteria. Astron. Astrophys. 527, A29. DOI. ADS.

Borrero, J.M., Kobel, P.: 2012, Inferring the magnetic field vector in the quiet Sun, II: Interpreting results from the inversion of Stokes profiles. Astron. Astrophys. 547, A89. DOI. ADS.

Borrero, J.M., Jafarzadeh, S., Schüssler, M., Solanki, S.K.: 2017, Solar magnetoconvection and small-scale dynamo. Recent developments in observation and simulation. Space Sci. Rev. 210, 275. DOI. ADS.

Centeno, R., Socas-Navarro, H., Lites, B., Kubo, M., Frank, Z., Shine, R., Tarbell, T., Title, A., Ichimoto, K., Tsuneta, S., Katsukawa, Y., Suematsu, Y., Shimizu, T., Nagata, S.: 2007, Emergence of small-scale magnetic loops in the quiet-sun internetwork. Astrophys. J. Lett. 666, L137. DOI. ADS.

Chae, J., Moon, Y.-J., Pevtsov, A.A.: 2004, Observational evidence of magnetic flux submergence in flux cancellation sites. Astrophys. J. Lett. 602, L65. DOI. ADS.

Cheung, M.C.M., Schüssler, M., Tarbell, T.D., Title, A.M.: 2008, Solar surface emerging flux regions: A comparative study of radiative MHD modeling and hinode SOT observations. Astrophys. J. 687, 1373. DOI. ADS.

Danilovic, S., vanNoort, M., Rempel, M.: 2016, Internetwork magnetic field as revealed by two-dimensional inversions. Astron. Astrophys. 593, A93. DOI. ADS.

Danilovic, S., Beeck, B., Pietarila, A., Schüssler, M., Solanki, S.K., Martínez Pillet, V., Bonet, J.A., del Toro Iniesta, J.C., Domingo, V., Barthol, P., Berkefeld, T., Gandorfer, A., Knölker, M., Schmidt, W., Title, A.M.: 2010, Transverse component of the magnetic field in the solar photosphere observed by SUNRISE. Astrophys. J. Lett. 723, L149. DOI. ADS.

De Pontieu, B.: 2002, High-resolution observations of small-scale emerging flux in the photosphere. Astrophys. J. 569, 474. DOI. ADS.

de Wijn, A.G., Stenflo, J.O., Solanki, S.K., Tsuneta, S.: 2009, Small-scale solar magnetic fields. Space Sci. Rev. 144, 275. DOI. ADS.

Fontenla, J.M., Avrett, E., Thuillier, G., Harder, J.: 2006, Semiempirical models of the solar atmosphere, I: The quiet- and active sun photosphere at moderate resolution. Astrophys. J. 639, 441. DOI. ADS.

Frutiger, C., Solanki, S.K., Fligge, M., Bruls, J.H.M.J.: 2000, Properties of the solar granulation obtained from the inversion of low spatial resolution spectra. Astron. Astrophys. 358, 1109. ADS.

Gingerich, O., Noyes, R.W., Kalkofen, W., Cuny, Y.: 1971, The Harvard-Smithsonian reference atmosphere. Solar Phys. 18, 347. DOI. ADS.

Gömöry, P., Beck, C., Balthasar, H., Rybák, J., Kučera, A., Koza, J., Wöhl, H.: 2010, Magnetic loop emergence within a granule. Astron. Astrophys. 511, A14. DOI. ADS.

Harvey, J.W., Branston, D., Henney, C.J., Keller, C.U., SOLIS and GONG Teams: 2007, Seething horizontal magnetic fields in the quiet solar photosphere. Astrophys. J. Lett. 659, L177. DOI. ADS.

Ishikawa, R., Tsuneta, S.: 2008, New form of emerging magnetic fields in plage regions. In: First Results from Hinode. ASP Conf. Ser. 397, 21. ADS.

Ishikawa, R., Tsuneta, S.: 2009, Comparison of transient horizontal magnetic fields in a plage region and in the quiet Sun. Astron. Astrophys. 495, 607. DOI. ADS.

Ishikawa, R., Tsuneta, S.: 2010, Spatial and temporal distributions of transient horizontal magnetic fields with deep exposure. Astrophys. J. Lett. 718, L171. DOI. ADS. 
Ishikawa, R., Tsuneta, S.: 2011, The relationship between vertical and horizontal magnetic fields in the quiet sun. Astrophys. J. 735, 74. DOI. ADS.

Jafarzadeh, S., Rouppe van der Voort, L., de la Cruz Rodríguez, J.: 2015, Magnetic upflow events in the quiet-sun photosphere, I: Observations. Astrophys. J. 810, 54. DOI. ADS.

Jafarzadeh, S., Solanki, S.K., Feller, A., Lagg, A., Pietarila, A., Danilovic, S., Riethmüller, T.L., Martínez Pillet, V.: 2013, Structure and dynamics of isolated internetwork Ca II H bright points observed by SUNRISE. Astron. Astrophys. 549, A116. DOI. ADS.

Jafarzadeh, S., Solanki, S.K., Lagg, A., Bellot Rubio, L.R., van Noort, M., Feller, A., Danilovic, S.: 2014, Inclinations of small quiet-Sun magnetic features based on a new geometric approach. Astron. Astrophys. 569, A105. DOI. ADS.

Jafarzadeh, S., Rutten, R.J., Solanki, S.K., Wiegelmann, T., Riethmüller, T.L., van Noort, M., Szydlarski, M., Blanco Rodríguez, J., Barthol, P., del Toro Iniesta, J.C., Gandorfer, A., Gizon, L., Hirzberger, J., Knölker, M., Martínez Pillet, V., Orozco Suárez, D., Schmidt, W.: 2017, Slender Ca II H fibrils mapping magnetic fields in the low solar chromosphere. Astrophys. J. Suppl. 229, 11. DOI. ADS.

Jin, C., Wang, J., Zhou, G.: 2009, The properties of horizontal magnetic elements in quiet solar intranetwork. Astrophys. J. 697, 693. DOI. ADS.

Kahil, F., Riethmüller, T.L., Solanki, S.K.: 2017, Brightness of solar magnetic elements as a function of magnetic flux at high spatial resolution. Astrophys. J. Suppl. 229, 12. DOI. ADS.

Khomenko, E.V., Collados, M., Solanki, S.K., Lagg, A., Trujillo Bueno, J.: 2003, Quiet-Sun inter-network magnetic fields observed in the infrared. Astron. Astrophys. 408, 1115. DOI. ADS.

Kubo, M., Shimizu, T.: 2007, Magnetic field properties of flux cancellation sites. Astrophys. J. 671, 990. DOI. ADS.

Lagg, A., Solanki, S.K., Doerr, H.-P., Martínez González, M.J., Riethmüller, T., Collados Vera, M., Schlichenmaier, R., Orozco Suárez, D., Franz, M., Feller, A., Kuckein, C., Schmidt, W., Asensio Ramos, A., Pastor Yabar, A., von der Lühe, O., Denker, C., Balthasar, H., Volkmer, R., Staude, J., Hofmann, A., Strassmeier, K., Kneer, F., Waldmann, T., Borrero, J.M., Sobotka, M., Verma, M., Louis, R.E., Rezaei, R., Soltau, D., Berkefeld, T., Sigwarth, M., Schmidt, D., Kiess, C., Nicklas, H.: 2016, Probing deep photospheric layers of the quiet Sun with high magnetic sensitivity. Astron. Astrophys. 596, A6. DOI. ADS.

Lin, H.: 1995, On the Distribution of the Solar Magnetic Fields. Astrophys. J. 446, 421. DOI. ADS.

Lin, H., Rimmele, T.: 1999, The granular magnetic fields of the quiet sun. Astrophys. J. 514, 448. DOI. ADS.

Lites, B.W.: 2002, Characterization of magnetic flux in the quiet sun. Astrophys. J. 573, 431. DOI. ADS.

Lites, B.W., Leka, K.D., Skumanich, A., Martinez Pillet, V., Shimizu, T.: 1996, Small-scale horizontal magnetic fields in the solar photosphere. Astrophys. J. 460, 1019. DOI. ADS.

Lites, B.W., Centeno, R., Kubo, M., Socas-Navarro, H.B.T., Frank, Z., Shine, R., Tarbell, T., Title, A., Ichimoto, K., Katsukawa, Y., Tsuneta, S., Suematsu, Y., Shimizu, T., Nagata, S.: 2008a, Hinode observations of flux emergence in quiet and active regions. In: Howe, R., Komm, R.W., Balasubramaniam, K.S., Petrie, G.J.D. (eds.) Subsurface and Atmospheric Influences on Solar Activity, Astron. Soc. Pac. Conf. Ser. 383, 71. ADS.

Lites, B.W., Kubo, M., Socas-Navarro, H., Berger, T., Frank, Z., Shine, R., Tarbell, T., Title, A., Ichimoto, K., Katsukawa, Y., Tsuneta, S., Suematsu, Y., Shimizu, T., Nagata, S.: 2008b, The horizontal magnetic flux of the quiet-sun internetwork as observed with the hinode spectro-polarimeter. Astrophys. J. 672, 1237. DOI. ADS.

Lites, B.W., Rempel, M., Borrero, J.M., Danilovic, S.: 2017, Are internetwork magnetic fields in the solar photosphere horizontal or vertical? Astrophys. J. 835, 14. DOI. ADS.

Livingston, W., Harvey, J.: 1971, The Kitt Peak Magnetograph, IV: 40-CHANNEL probe and the detection of weak photospheric fields. In: Howard, R. (ed.) Solar Magnetic Fields, IAU Symp. 43, 51. ADS.

Livingston, W.C., Harvey, J.: 1975, A new component of solar magnetism - the inner network fields. Bull. Am. Astron. Soc. 7, 346. ADS.

Martin, S.F.: 1988, The identification and interaction of network, intranetwork, and ephemeral-region magnetic fields. Solar Phys. 117, 243. DOI. ADS.

Martínez González, M.J., Bellot Rubio, L.R.: 2009, Emergence of small-scale magnetic loops through the quiet solar atmosphere. Astrophys. J. 700, 1391. DOI. ADS.

Martínez González, M.J., Collados, M., Ruiz Cobo, B., Solanki, S.K.: 2007, Low-lying magnetic loops in the solar internetwork. Astron. Astrophys. 469, L39. DOI. ADS.

Martínez González, M.J., Collados, M., Ruiz Cobo, B., Beck, C.: 2008, Internetwork magnetic field distribution from simultaneous $1.56 \mu \mathrm{m}$ and $630 \mathrm{~nm}$ observations. Astron. Astrophys. 477, 953. DOI. ADS.

Martínez González, M.J., Pastor Yabar, A., Lagg, A., Asensio Ramos, A., Collados, M., Solanki, S.K., Balthasar, H., Berkefeld, T., Denker, C., Doerr, H.P., Feller, A., Franz, M., González Manrique, S.J., Hofmann, A., Kneer, F., Kuckein, C., Louis, R., von der Lühe, O., Nicklas, H., Orozco, D., Rezaei, R., Schlichenmaier, R., Schmidt, D., Schmidt, W., Sigwarth, M., Sobotka, M., Soltau, D., Staude, J., 
Strassmeier, K.G., Verma, M., Waldman, T., Volkmer, R.: 2016, Inference of magnetic fields in the very quiet Sun. Astron. Astrophys. 596, A5. DOI. ADS.

Martínez Pillet, V., del Toro Iniesta, J.C., Álvarez-Herrero, A., Domingo, V., Bonet, J.A., González Fernández, L., López Jiménez, A., Pastor, C., Gasent Blesa, J.L., Mellado, P., Piqueras, J., Aparicio, B., Balaguer, M., Ballesteros, E., Belenguer, T., Bellot Rubio, L.R., Berkefeld, T., Collados, M., Deutsch, W., Feller, A., Girela, F., Grauf, B., Heredero, R.L., Herranz, M., Jerónimo, J.M., Laguna, H., Meller, R., Menéndez, M., Morales, R., Orozco Suárez, D., Ramos, G., Reina, M., Ramos, J.L., Rodríguez, P., Sánchez, A., Uribe-Patarroyo, N., Barthol, P., Gandorfer, A., Knoelker, M., Schmidt, W., Solanki, S.K., Vargas Domínguez, S.: 2011, The Imaging Magnetograph eXperiment (IMaX) for the Sunrise Balloon-Borne Solar Observatory. Solar Phys. 268, 57. DOI. ADS.

Meunier, N., Solanki, S.K., Livingston, W.C.: 1998, Infrared lines as probes of solar magnetic features, XIII: The relative flux in weak and strong quiet-sun magnetic fields. Astron. Astrophys. 331, 771. ADS.

Nordlund, A., Stein, R.F., Asplund, M.: 2009, Solar surface convection. Living Rev. Solar Phys. 6, 2. DOI. ADS.

Orozco Suárez, D., Bellot Rubio, L.R.: 2012, Analysis of quiet-sun internetwork magnetic fields based on linear polarization signals. Astrophys. J. 751, 2. DOI. ADS.

Orozco Suárez, D., Bellot Rubio, L.R., del Toro Iniesta, J.C., Tsuneta, S., Lites, B.W., Ichimoto, K., Katsukawa, Y., Nagata, S., Shimizu, T., Shine, R.A., Suematsu, Y., Tarbell, T.D., Title, A.M.: 2007, Quietsun internetwork magnetic fields from the inversion of hinode measurements. Astrophys. J. Lett. 670, L61. DOI. ADS.

Roudier, T., Eibe, M.T., Malherbe, J.M., Rieutord, M., Mein, P., Mein, N., Faurobert, M.: 2001, Temporal height properties of the exploding granules. Astron. Astrophys. 368, 652. DOI. ADS.

Sánchez Almeida, J., Martínez González, M.: 2011, The magnetic fields of the quiet sun. In: Kuhn, J.R., Harrington, D.M., Lin, H., Berdyugina, S.V., Trujillo-Bueno, J., Keil, S.L., Rimmele, T. (eds.) Solar Polarization 6, Astron. Soc. Pac. Conf. Ser. 437, 451. ADS.

Schrijver, C.J., Title, A.M., van Ballegooijen, A.A., Hagenaar, H.J., Shine, R.A.: 1997, Sustaining the quiet photospheric network: The balance of flux emergence, fragmentation, merging, and cancellation. Astrophys. J. 487, 424. DOI. ADS.

Solanki, S.K.: 1987, The photospheric layers of solar magnetic flux tubes. PhD thesis, No. 8309, ETH, Zürich. ADS.

Solanki, S.K.: 2009, Photospheric magnetic field: Quiet sun. In: Berdyugina, S.V., Nagendra, K.N., Ramelli, R. (eds.) Solar Polarization 5: In Honor of Jan Stenflo, Astron. Soc. Pac. Conf. Ser. 405, 135. ADS.

Solanki, S.K., Barthol, P., Danilovic, S., Feller, A., Gandorfer, A., Hirzberger, J., Riethmüller, T.L., Schüssler, M., Bonet, J.A., Martínez Pillet, V., del Toro Iniesta, J.C., Domingo, V., Palacios, J., Knölker, M., Bello González, N., Berkefeld, T., Franz, M., Schmidt, W., Title, A.M.: 2010, SUNRISE: Instrument, mission, data, and first results. Astrophys. J. Lett. 723, L127. DOI. ADS.

Stein, R.F., Nordlund, Å.: 2006, Solar small-scale magnetoconvection. Astrophys. J. 642, 1246. DOI. ADS.

Stenflo, J.O.: 1971, The interpretation of magnetograph results: The formation of absorption lines in a magnetic field. In: Howard, R. (ed.) Solar Magnetic Fields, IAU Symp. 43, 101, Reidel, Dordrecht.

Stenflo, J.O.: 2008, Solar magnetic fields. J. Astrophys. Astron. 29, 19. DOI. ADS.

Stenflo, J.O.: 2010, Distribution functions for magnetic fields on the quiet Sun. Astron. Astrophys. 517, A37. DOI. ADS.

Stenflo, J.O.: 2012, Angular distribution of quiet-sun magnetic fields. In: Sekii, T., Watanabe, T., Sakurai, T. (eds.) Hinode-3: The 3rd Hinode Science Meeting, Astron. Soc. Pac. Conf. Ser. 454, 33. ADS.

Stenflo, J.O.: 2013, Horizontal or vertical magnetic fields on the quiet Sun. Angular distributions and their height variations. Astron. Astrophys. 555, A132. DOI. ADS.

Tsuneta, S., Ichimoto, K., Katsukawa, Y., Lites, B.W., Matsuzaki, K., Nagata, S., Orozco Suárez, D., Shimizu, T., Shimojo, M., Shine, R.A., Suematsu, Y., Suzuki, T.K., Tarbell, T.D., Title, A.M.: 2008, The magnetic landscape of the sun's polar region. Astrophys. J. 688, 1374. DOI. ADS.

Wittmann, A.: 1974, Computation and observation of zeeman multiplet polarization in fraunhofer lines, II: Computation of stokes parameter profiles. Solar Phys. 35, 11. DOI. ADS. 